\title{
Las TIC en los procesos de enseñanza-aprendizaje desarrollados por maestros tutores de Educación Primaria en la Región de Murcia
}

\section{ICT in the teaching-learning processes developed by Primary Education tutors in the Region of Murcia}

\author{
David Castillo López \\ Universidad de Murcia (España) \\ Davicas95@gmail.com
}

Recibido: $11 / 06 / 2020$

Aceptado: $15 / 09 / 2020$

Publicado: 01/12/2020

\section{RESUMEN}

Las tecnologías de la información y la comunicación (TIC) tienen un papel esencial en la sociedad. En consecuencia, en el ámbito educativo, se ha incrementado la presencia de dispositivos tecnológicos y de contenidos orientados al fomento de la competencia digital de los estudiantes. Para alcanzar este propósito, es necesario que el profesorado incorpore, en el proceso de enseñanza-aprendizaje, recursos digitales y metodologías que promuevan en el alumnado el aprendizaje y el desarrollo de habilidades digitales. Con este estudio se pretende conocer el uso que hacen de las TIC los maestros tutores de Educación Primaria de colegios públicos de la Región de Murcia. Así, se analizan, principalmente, cuáles son los recursos digitales y las estrategias metodológicas que suelen emplear en el proceso de enseñanza-aprendizaje estos docentes. Para ello, se ha llevado a cabo una investigación, de tipo descriptiva y desde la metodología cuantitativa, en la que la recogida de datos se ha realizado a través de un cuestionario elaborado para este estudio, que fue contestado por un total de 133 docentes. Los resultados obtenidos revelan que, aunque se han llegado a introducir metodologías activas, los docentes encuestados no utilizan con frecuencia en el proceso de enseñanza-aprendizaje recursos tecnológicos innovadores ni metodologías emergentes en el uso de las TIC.

\section{PALABRAS CLAVE}

TIC; enseñanza; metodología; aprendizaje; recursos.

\section{ABSTRACT}

Information and communication technologies (ICT) play an essential role in society. Consequently, in the educational field, the presence of technological devices and contents aimed at promoting the digital competence of students has increased. To achieve this purpose, it is necessary for teachers to incorporate, in the teaching-learning process, digital resources and methodologies that promote learning and digital skills development in students. The aim of this research is to find out the use of ICT by Primary Education tutors in public schools in the Region of Murcia. Thus, we analyze, mainly, which are the digital resources and the methodological strategies that these teachers usually use in the teaching-learning process. To this end, a descriptive investigation is carried out from the quantitative methodology, in which the collection of data has been carried out through a questionnaire prepared for this study, which was answered by a total of 133 teachers. The results obtained reveal that, although active methodologies have been introduced, the teachers surveyed do not often use innovative technological resources or emerging methodologies in the use of ICT into the teaching-learning process.

\section{KEYWORDS}

ICT; teaching; methodology; learning; resources. 


\section{CITA RECOMENDADA}

Castillo, D. (2020). Las TIC en los procesos de enseñanza-aprendizaje desarrollados por maestros tutores de Educación Primaria en la Región de Murcia. Revista Interuniversitaria de Investigación en Tecnología Educativa, 9, 1-14. https://doi.org/10.6018/riite.432061

\section{Principales aportaciones del artículo y futuras líneas de investigación:}

- Conocer el uso que hacen de las TIC, en el proceso de enseñanza-aprendizaje, los maestros tutores de Educación Primaria de los colegios públicos de la Región de Murcia participantes en la investigación.

- Identificar los recursos digitales y tecnológicos, así como las metodologías activas, que suelen utilizar con más frecuencia los maestros tutores de Educación Primaria participantes en este estudio.

- Líneas futuras de investigación: ampliar esta investigación analizando los recursos digitales y los dispositivos tecnológicos con los que cuentan los centros educativos de Educación Primaria; realizar estudios similares con una muestra mayor; y desarrollar investigaciones con técnicas cualitativas para enriquecer, comparar y analizar con mayor profundidad los resultados de este estudio.

\section{INTRODUCCIÓN}

El empleo de las TIC en el ámbito educativo se ha generalizado los últimos años. En consecuencia, se han ampliado los espacios formativos, se han promovido nuevas formas de interacción entre los estudiantes y el personal docente, se ha favorecido el desarrollo de una enseñanza más personalizada, se ha contribuido a la mejora de la comunicación entre los distintos agentes educativos, se han incrementado las posibilidades para alcanzar la inclusión social, se ha facilitado la accesibilidad a los contenidos y se han promovido las inteligencias múltiples de los alumnos, entre otros aspectos (Cabero \& Martínez, 2019; Martínez et al., 2018).

Numerosos estudios, como el llevado a cabo por Domingo \& Marquès (2011), revelan que el uso de las TIC en el proceso de enseñanza-aprendizaje puede aportar ventajas como las siguientes: facilitar el logro de los objetivos, el desarrollo del proceso de enseñanza y la comprensión de los contenidos; promover la renovación desde el punto de vista metodológico; e incrementar la atención de los estudiantes, entre otras.

Las TIC se han ido introduciendo, pues, en los currículos de enseñanza y se ha incrementado, en consecuencia, el empleo de herramientas tecnológicas en los centros educativos. Sin embargo, a pesar del desarrollo de acciones como, por ejemplo, aumentar la presencia de los medios tecnológicos e implantar planes concretos, no se están transformando siempre las dinámicas pedagógicas y, en consecuencia, diversos estudios concluyen que la introducción de las TIC no siempre está dando como resultado un aumento de los aprendizajes de los alumnos (Cabero \& Ruiz, 2018).

Al respecto, es importante tener en cuenta que, según Granados (2015), la utilización de las TIC en el proceso de enseñanza-aprendizaje implica que el profesorado no solo esté capacitado para utilizar las herramientas más adecuadas, sino que, a su vez, este último se tiene que formar y debe actualizar las estrategias metodológicas a la hora de introducir las TIC en el aula. En este sentido, los docentes deben diseñar y desarrollar dinámicas que contribuyan a la innovación y a la mejora del proceso de aprendizaje del estudiante, pero deben tener en cuenta que las TIC, por sí solas, no son la solución, ya que facilitar a los estudiantes una tableta o a un libro digital no constituye un proceso de innovación desde el punto de vista metodológico (Mato \& Álvarez, 2015).

En consecuencia, debemos considerar que el simple uso de los recursos digitales no se traduce en una mejora del proceso educativo, ya que la pieza fundamental para conseguir buenos resultados se basa en el diseño de tareas didácticas adecuadas (Nieto \& Marquès, 2015). La incorporación de las TIC en el aula exige, según Díaz-Barriga (2013), dotar a estas últimas de un fin didáctico en el momento de ponerlas en práctica, de forma que el alumnado aprenda a través de su utilización. El profesorado ejerce un papel crucial en este proceso de planificación 
didáctica, ya que debe estar capacitado para introducir estrategias metodológicas que faciliten el aprovechamiento de las TIC en el proceso de enseñanza (Hernández, 2017).

Sin embargo, en el contexto educativo, es común pensar que las TIC, por sí mismas, tienen la capacidad de mejorar el proceso de enseñanza, lo que significa que no se presta atención a elementos relacionados con la organización y planificación pedagógica del proceso de enseñanza (Moreno, 2018). Así, uno de los errores más relevantes que se han cometido al incorporar las TIC en la enseñanza formal, según Llorente et al. (2015), ha sido llegar a percibir los recursos tecnológicos como aspectos que son independientes del resto de elementos que integran el currículo.

En el proceso de enseñanza-aprendizaje, a la hora de introducir las TIC es necesario, pues, la capacitación del profesorado en el uso de las mismas y el desarrollo de un proceso de organización y planificación que tenga en cuenta aspectos como los contenidos, el alumnado y el contexto, así como la puesta en práctica de metodologías que faciliten el uso adecuado de los recursos tecnológicos (Hernández, 2017; Martínez et al. 2018).

Martínez et al. (2018) afirman que los docentes deben asumir nuevos roles, siendo conveniente un cambio de valores en relación al desarrollo y organización del proceso de enseñanza. Esto implica la predisposición por parte del profesorado, así como la formación del mismo en el empleo de las TIC y en la aplicación de las estrategias metodológicas más adecuadas en el aula.

En la etapa de Educación Primaria, se ha generalizado el uso de recursos tecnológicos, como los ordenadores o la pizarra digital interactiva. A su vez, diversas investigaciones evidencian los beneficios que supone el uso de recursos digitales emergentes como, por ejemplo, la robótica educativa, las tabletas, las aplicaciones multimedia, las redes sociales, la realidad aumentada o la realidad virtual en el ámbito educativo (Aznar et al. 2018; Céspedes, 2017; Bravo \& Forero, 2012; Fuentes et al., 2019; Recio, 2019; López et al., 2020).

Por otro lado, diferentes investigaciones en el ámbito educativo han demostrado los beneficios que aporta en el proceso educativo el empleo de estrategias metodológicas activas a la hora de utilizar las TIC, como son la gamificación, el aprendizaje basado en proyectos, el flipped classroom, el aprendizaje basado en juegos, el aprendizaje basado en problemas o el aprendizaje cooperativo, entre otras (Boyle, 2011; González \& Zariquiey, 2012; Rekalde \& García, 2015; Sánchez et al. 2017; Sebastian et al., 2013; y Villena et al., 2018).

Por otro lado, se debe tener en cuenta que la Educación Primaria constituye una de las etapas cruciales en el desarrollo cognitivo, afectivo y social del individuo. Durante este periodo, los maestros tutores de los distintos cursos ejercen un papel crucial, ya que son los docentes que mayor tiempo atienden a los estudiantes. En consecuencia, en el presente estudio se seleccionó como población al conjunto de docentes que ejercen como tutores de Educación Primaria en los centros educativos de titularidad pública de la Región de Murcia.

En suma, el propósito de este trabajo es identificar cuáles son los recursos TIC que, en términos generales, suelen emplear con mayor frecuencia los docentes tutores de Primaria en los colegios de titularidad pública de la Región de Murcia y, a su vez, conocer cuáles son las estrategias metodológicas que utilizan los mismos al emplear las TIC en el proceso de enseñanza-aprendizaje. Del mismo modo, se pretende averiguar cuál es la percepción que tienen estos maestros con respecto al empleo de metodologías activas al introducir las TIC en el proceso de enseñanza.

\section{MÉTODO}

\subsection{Objetivos}

Los objetivos generales y específicos de la investigación son los que se presentan a continuación. 
Objetivo general 1: Conocer el uso que hacen de los recursos tecnológicos y digitales los maestros que ejercen como tutores de Educación Primaria en colegios públicos de la Región de Murcia. Se concreta en los siguientes objetivos específicos:

- Conocer los recursos digitales y tecnológicos que emplean los tutores.

- Identificar la frecuencia con la que los tutores hacen uso de los recursos digitales y tecnológicos.

Objetivo general 2: Conocer el uso de las estrategias metodológicas apoyadas en el uso de las TIC por parte de los maestros que ejercen como tutores de Educación Primaria en colegios públicos de la Región de Murcia. Se concreta en los siguientes objetivos específicos:

- Identificar las estrategias metodológicas que conocen y emplean los tutores.

- Conocer la percepción que tienen los tutores acerca del desarrollo de estrategias metodológicas activas al hacer uso de las TIC.

\subsection{Enfoque de la investigación}

Con la presente investigación se pretende conocer y analizar los recursos digitales y las estrategias metodológicas enriquecidas con el uso de las TIC que emplean los maestros tutores de Educación Primaria en el proceso de enseñanza-aprendizaje. Para ello, partiendo de la naturaleza y del tratamiento de los datos, este estudio se lleva a cabo siguiendo la metodología cuantitativa, en la que se realizan mediciones numéricas y se analizan estadísticamente los resultados (Hernández et al., 2014).

La investigación es de tipo descriptiva, ya que se centra en describir y proporcionar información sobre hechos (Cardona, 2002) y se desarrolla desde el enfoque no experimental. Para realizar la recogida de datos, a partir de los objetivos de la presente investigación, se diseñó un cuestionario que, posteriormente, fue validado por tres expertos en Tecnología Educativa.

\subsection{Participantes}

La población que se toma como objeto de estudio de esta investigación la constituyen los maestros que ejercen como tutores de Educación Primaria en los colegios públicos de la Región de Murcia. Así, la muestra participante en el estudio la forman un total de 133 maestros tutores de Primaria de distintos centros educativos públicos de esta Comunidad Autónoma. Cabe destacar que el $72,9 \%$ de los encuestados son mujeres y que la edad del total de participantes oscila entre los 24 y los 64 años, siendo los 41 años la edad media. La mayoría de estos maestros tiene más de 10 años de experiencia docente (63,9\%), el 13,5\% cuenta con una experiencia de entre 5 y 10 años, el 14,3\% posee una experiencia de entre 1 y 5 años y el 8,3\% tiene una experiencia inferior a un año.

\subsection{Instrumento de recogida de datos}

El instrumento que se utilizó para recoger los datos fue un cuestionario construido con la herramienta Google Forms. Para el diseño del mismo, se elaboraron preguntas a partir de los objetivos de este estudio. El cuestionario lo validaron tres expertos en Tecnología Educativa y, posteriormente, fue enviado a los correos electrónicos de los colegios públicos que imparten la etapa de Educación Primaria en la Región de Murcia, incluyendo en el mensaje enviado un enlace con acceso directo al cuestionario. Más adelante, El mismo se difundió a través de Twitter y WhatsApp. Los cuestionarios fueron contestados entre los meses de marzo y abril del año 2020.

En el cuestionario se incluyeron 15 cuestiones o ítems, aunque en dos de las cuestiones de respuesta cerrada se introdujeron más ítems diferentes, por lo que se podría hablar de un total de 38 ítems. De todos ellos, 34 fueron de respuesta cerrada, 2 de respuesta semiabierta y otros 2 de respuesta abierta. Estas cuestiones se distribuyeron en las siguientes secciones:

- Apartado 1. Presentación y preguntas previas. Se incluye el título, la presentación del cuestionario y cuestiones relacionadas con el sexo, la edad y los años de experiencia en la docencia de los encuestados. 
- Apartado 2. Formación y empleo de recursos TIC. En esta sección se incluyen cuestiones relacionadas con la formación académica, la formación en el empleo de las TIC, el dominio de las TIC y la percepción en relación a la importancia de introducir las TIC en el aula. Asimismo, se recoge una pregunta amplia, que integra otras cuestiones o ítems, para identificar la frecuencia de uso de recursos tecnológicos concretos.

- Apartado 3. Estrategias metodológicas y TIC. En este apartado se recoge una pregunta, que incluye diferentes ítems, con la que se pretende conocer la frecuencia en la que se utilizan determinadas metodologías activas al usar las TIC en el proceso de enseñanzaaprendizaje. A su vez, se incluyen cuestiones para conocer la percepción que tienen los participantes en relación al uso de metodologías activas al emplear las TIC.

\subsection{Tratamiento y análisis de datos}

Para llevar a cabo el proceso de recogida de datos, en primer lugar, el cuestionario fue cerrado desde la herramienta Google Forms. Seguidamente, se generó una hoja de cálculo de Excel. El paso siguiente fue depurar y codificar los resultados obtenidos, asignando valores numéricos a las respuestas recogidas.

Posteriormente, los datos fueron analizados cuantitativamente a través del programa SPPS, a partir de cual se recogió y organizó la información estadísticamente, calculando las frecuencias y los porcentajes de las variables estudiadas. A partir de los datos estadísticos obtenidos, se elaboraron gráficos de frecuencias que facilitasen la interpretación y el análisis de los resultados.

A su vez, desde el programa SPSS, se ha llevado a cabo un análisis correlacional con el objetivo de identificar posibles diferencias estadísticamente significativas entre las variables analizadas.

Por otro lado, en las dos cuestiones de respuesta abierta se llevó a cabo un análisis cuantitativo de los datos, con el fin de calcular la frecuencia con la que los maestros respondían afirmativamente o negativamente a las cuestiones, estableciendo, a través del programa SPSS las etiquetas "responde" y "no responde" y asignándole valores numéricos a las mismas. Seguidamente, debido a la poca abundancia de respuestas abiertas, así como a la concreción de las mismas, se decidió describir y analizar, mediante un recuento manual, el número de veces en el que aparecían repetidas las respuestas dadas. De esta manera, se han categorizado las respuestas dadas y se ha indicado la frecuencia con la que aparecían las mismas.

A partir de los resultados recogidos, se ha realizado un análisis descriptivo de las diferentes variables estudiadas y, posteriormente, se han extraído unas conclusiones para dar respuesta a los objetivos de este estudio.

\section{RESULTADOS}

\subsection{Formación, importancia y nivel de dominio de las TIC}

La mayoría de los participantes en el estudio $(88,7 \%)$ ha recibido formación en relación al empleo de las TIC a nivel pedagógico. Asimismo, la mayoría de los encuestados considera que las TIC tienen mucha importancia (54,9\%) o bastante importancia $(43,6 \%)$ en el ámbito educativo. Solo el $1,5 \%$ cree que las TIC tienen poca importancia en el proceso de enseñanza-aprendizaje.

En relación a las habilidades en el uso de las TIC (Figura 1), la mayoría de los docentes cree tener un nivel de dominio bueno $(59,4 \%)$, mientras que el $18,8 \%$ considera que su nivel es avanzado. El resto afirma que su nivel es bajo. 


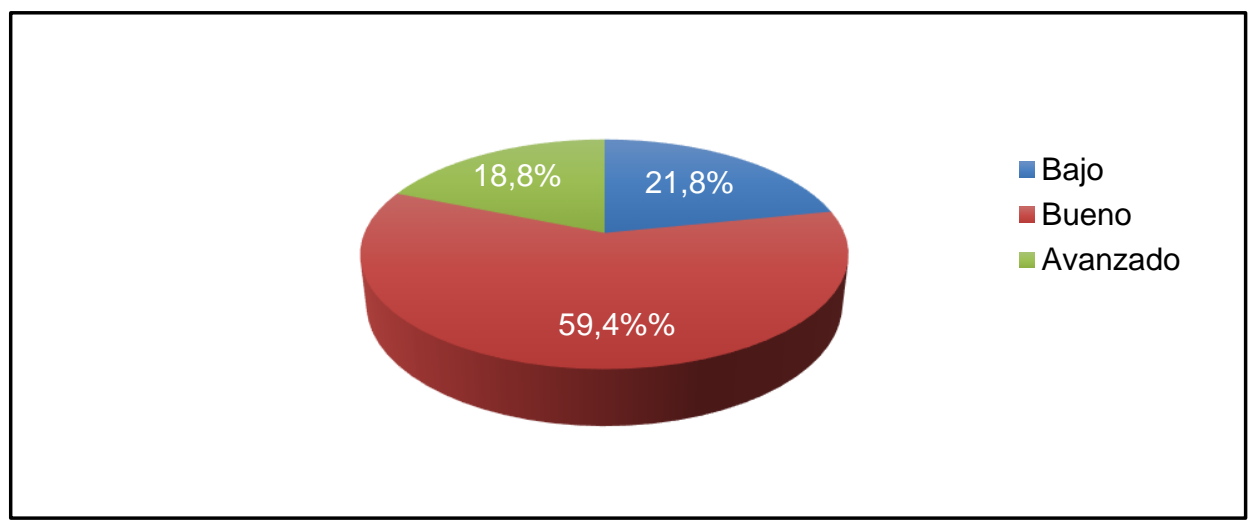

Figura 1. Nivel de dominio de las TIC

\subsection{Recursos digitales}

Los recursos TIC que presentan una mayor frecuencia de uso (Figura 2) son el ordenador y los proyectores, ya que en torno al $80 \%$ de los maestros encuestados incorpora los mismos siempre o casi siempre en el proceso de enseñanza-aprendizaje.

Se observa, a su vez, que recursos como las pizarras digitales interactivas, las aplicaciones ofimáticas y los recursos impresos de internet son utilizados casi siempre o siempre por cerca del $70 \%$ de los docentes participantes en este estudio. Destaca, además, la alta frecuencia de uso del libro de texto digital y de las aplicaciones de internet, que son empleados casi siempre o siempre por más del $60 \%$ de los maestros.

Por otro lado, se observa que los recursos tecnológicos más innovadores, como son la robótica educativa, la realidad virtual y la aumentada, son los usados con menor frecuencia por los maestros participantes en el estudio. En concreto, la robótica es el menos empleado, ya que el $76,7 \%$ de los encuestados no la usa nunca, el $12,8 \%$ casi nunca y tan solo el $10,5 \%$ la ha utilizado con cierta frecuencia.

Al respecto, podemos apreciar que, en relación al uso de recursos TIC, los maestros tutores incorporan con mayor frecuencia en el proceso de enseñanza-aprendizaje los recursos más convencionales o tradicionales en el ámbito tecnológico y digital.

Por otro lado, los resultados reflejan que la mayoría de los docentes encuestados no suelen emplear las tabletas con mucha frecuencia, ya que el $42,1 \%$ señala que nunca utiliza estos recursos en el proceso de enseñanza-aprendizaje, el $13,5 \%$ no las emplea casi nunca y el $26,3 \%$ las utiliza a veces. Tan solo el 18,1\% emplea estos dispositivos casi siempre o siempre.

Tampoco son utilizados con mucha frecuencia los teléfonos móviles inteligentes, las redes sociales, las wikis y las aplicaciones basadas en juegos por parte de los maestros encuestados, ya que cerca del $60 \%$ no introduce los mismos en el proceso educativo nunca o casi nunca. No obstante, recursos como las aplicaciones gamificadoras, los blogs o las aplicaciones de internet o de geolocalización si suelen ser utilizadas con cierta frecuencia, ya que más de la mitad de los docentes encuestados las emplea a veces, casi siempre o siempre. 


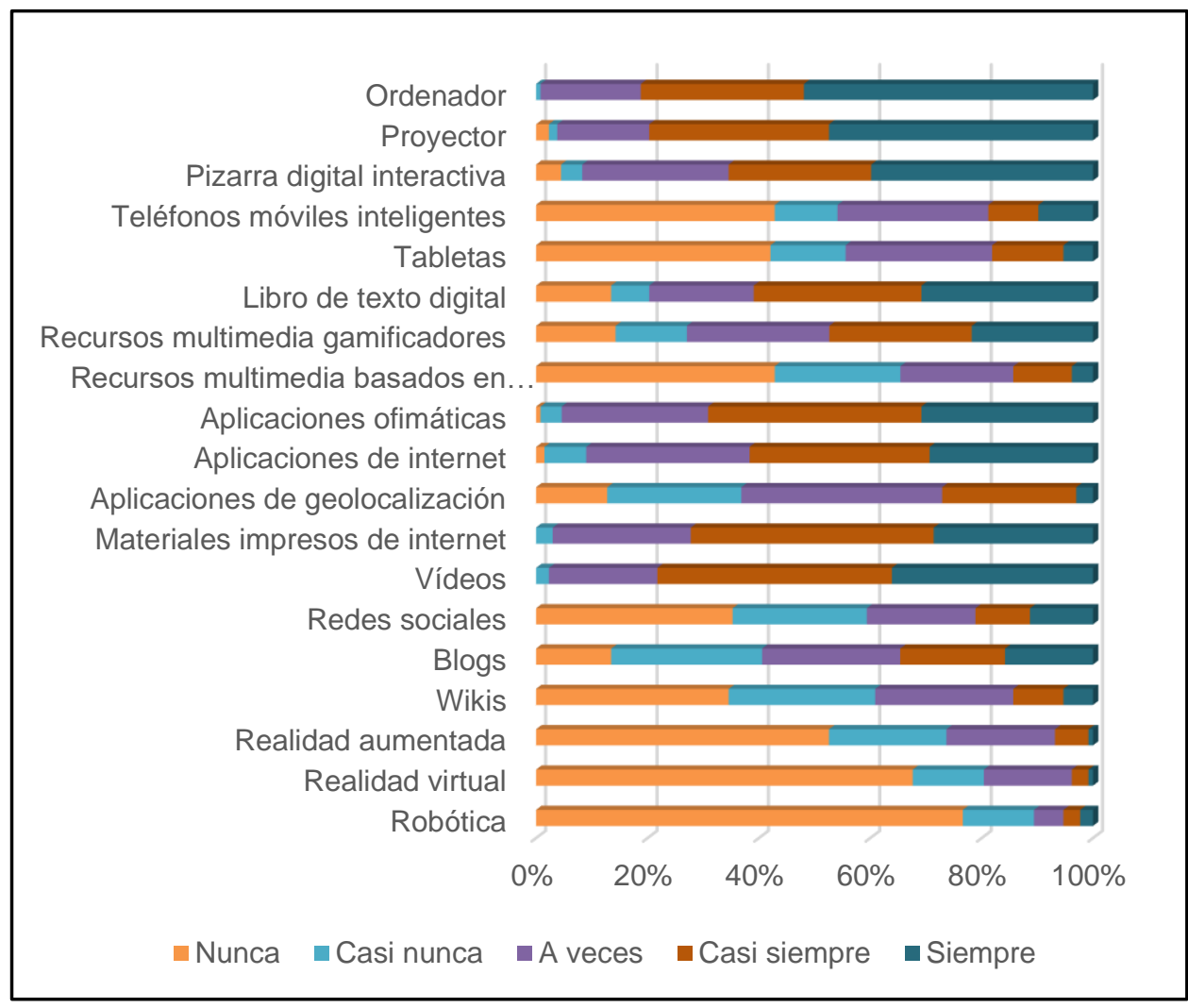

Figura 2. Recursos digitales y frecuencia de uso

Cabe destacar que, tras realizar análisis correlacionales (Tau-b de Kendall), se han observado diferencias estadísticamente significativas entre el uso de los recursos TIC y la experiencia docente, así como con respecto al nivel de dominio de las TIC.

En concreto, los resultados de este análisis revelan que, de los maestros encuestados, los que cuentan con más de 20 años de experiencia docente incorporan las tabletas en el aula con menor frecuencia que aquellos cuya experiencia en este ámbito es inferior a los 20 años (significación aproximada de 0,039).

Asimismo, los docentes con una experiencia docente superior a los 20 años emplean en el proceso de enseñanza-aprendizaje los recursos multimedia gamificadores con menos frecuencia que los docentes que cuentan con menos de 20 años de experiencia en este ámbito (significación aproximada de 0,003 ).

Por otro lado, con los resultados del análisis correlacional se observa que aquellos docentes que afirman poseer un nivel de dominio de las TIC bueno o avanzado incorporan en el proceso de enseñanza-aprendizaje las pizarras digitales interactivas, las aplicaciones ofimáticas, los recursos multimedia gamificadores, las aplicaciones de internet, los blogs, las aplicaciones de geolocalización y la realidad aumentada con más frecuencia que aquellos que consideran tener un nivel de dominio de las TIC bajo (significación aproximada de 0,000). Esta tendencia se repite con recursos como las tabletas (significación aproximada de 0,012 ), los teléfonos móviles inteligentes (significación aproximada de 0,015), los recursos multimedia basados en juegos (significación aproximada de 0,003 ), las wikis (significación aproximada de 0,039 ) y la robótica (significación aproximada de 0,013).

Con respecto a las respuestas dadas a la pregunta abierta, en la que se planteaba a los docentes la posibilidad de citar otros recursos digitales que utilizaran en el proceso de enseñanza-aprendizaje, solo el 20,5\% aportó ejemplos de recursos o herramientas. Los recursos que más veces se mencionaron fueron Google Classroom (7 veces), Genially (5 veces), Canva (4 veces), Google Suite for Education (4 veces), Symbaloo (3 veces), Scratch (3 veces), Padlet (2 veces) y Code (2 veces). 


\subsection{Estrategias metodológicas activas}

Se observa que, de las estrategias metodológicas planteadas (Figura 3), el aprendizaje cooperativo es la empleada con mayor frecuencia por parte de los docentes encuestados. Seguidamente, las utilizadas con más frecuencia son el aprendizaje basado en juegos y el aprendizaje basado en proyectos, mientras que la implementada con menor frecuencia es el flipped classroom.

En concreto, gran mayoría de los maestros $(91,8 \%)$ pone en práctica el aprendizaje cooperativo siempre, casi siempre o a veces en el proceso de enseñanza-aprendizaje. Por otro lado, el aprendizaje basado en juegos es usado a veces por el $45,1 \%$ de los encuestados, mientras que el $36,9 \%$ lo emplean siempre o casi siempre en el aula. El aprendizaje basado en proyectos, por su parte, es empleado casi siempre o siempre por el $27,8 \%$ y a veces por el $45,1 \%$, por lo que se considera que la frecuencia de uso de estas estrategias es alta.

El aprendizaje basado en problemas, por su parte, no es una estrategia con una alta frecuencia de uso por parte de los tutores participantes, ya que el $42,9 \%$ la utiliza a veces y el $30,1 \%$ no la emplea casi nunca. La gamificación, por otro lado, también es una tendencia metodológica cuya frecuencia de uso es bastante baja, ya que casi la mitad de los maestros encuestados (42,8\%) afirma no emplearla casi nunca o nunca y cerca del $34,6 \%$ la aplica en el aula a veces.

El flipped classroom obtiene los resultados más bajos en relación a la frecuencia de uso, ya que la mayoría de los encuestados $(63,1 \%)$ no la ha pone en práctica en el proceso de enseñanza-aprendizaje casi nunca o nunca. Solo cerca del $5,6 \%$ de los encuestados la implementa en el aula siempre o casi siempre.

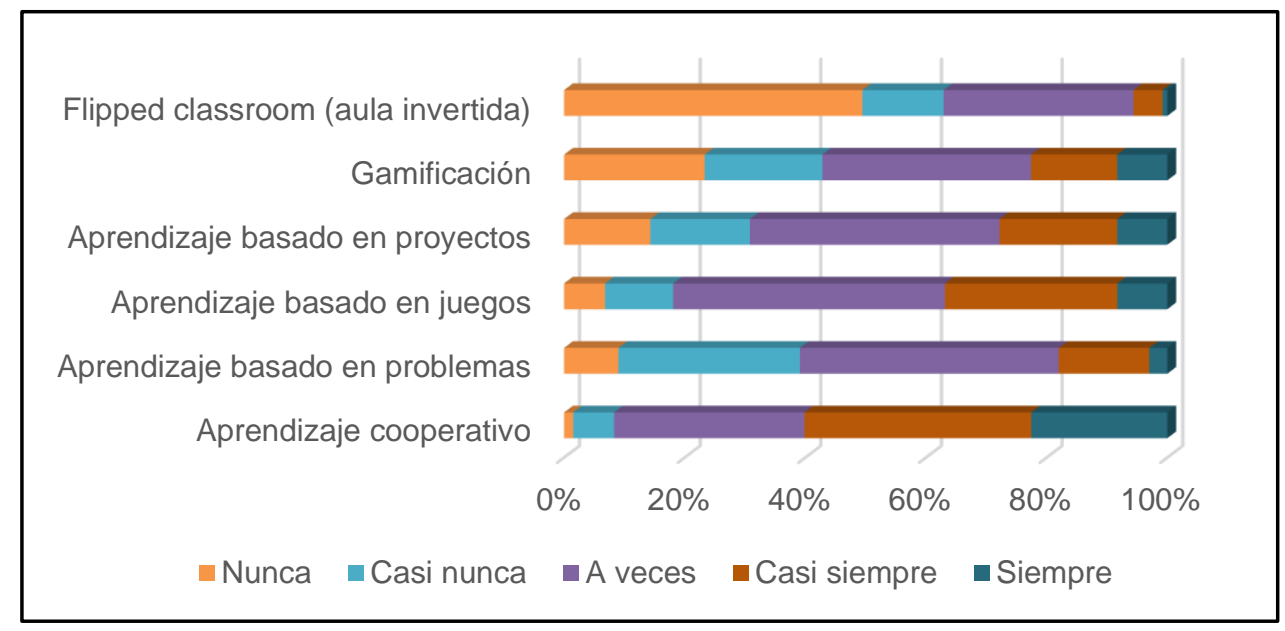

Figura 3. Estrategias metodológicas y frecuencia de uso

Cabe destacar que, tras realizar un análisis correlacional (Tau-b de Kendall), se observa que los maestros con 10 o más años de experiencia docente utilizan en el proceso de enseñanzaaprendizaje la gamificación con menos frecuencia que aquellos cuya experiencia es inferior a los 10 años (significación aproximada de 0,000). A su vez, los que afirman poseer un nivel de dominio de las TIC bueno o avanzado implementan con más frecuencia el flipped classroom y la gamificación que aquellos que poseen un nivel de dominio bajo (significación aproximada de 0,000).

Por otra parte, en la pregunta abierta en la que se planteaba la posibilidad de mencionar otras estrategias metodológicas que utilizaran en el aula y que no se hubieran propuesto en el cuestionario, solo el $25,6 \%$ de los maestros encuestados aportó estrategias diferentes. Las citadas con mayor frecuencia fueron la lección magistral (3 veces), el aprendizaje basado en las tareas (3 veces) y los rincones de trabajo (3 veces). 


\subsection{Percepción sobre el uso de estrategias metodológicas activas al emplear las TIC}

A la hora de concretar qué elemento es más importante tener en cuenta al emplear las TIC en el aula con el alumnado en una de las preguntas semiabiertas (Figura 4), la mayoría de los docentes participantes $(60,1 \%)$ cree que tanto el recurso digital empleado como la estrategia metodológica implementada tienen la misma importancia. No obstante, casi uno de cada tres docentes $(32,3 \%)$ considera que la estrategia metodológica es el elemento más importante, mientras que el $5,3 \%$ cree que es más relevante recurso digital utilizado. El resto de docentes encuestados considera que son más importantes otros elementos distintos a los anteriores.

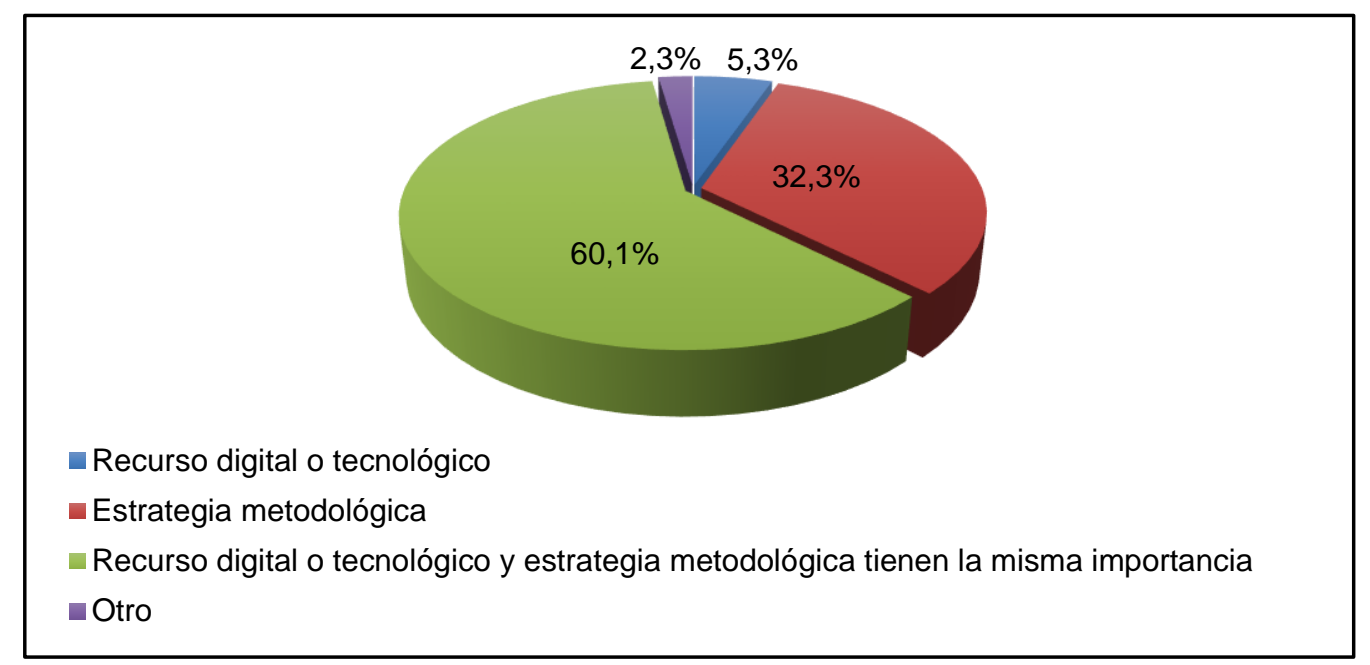

Figura 4. Elementos más relevantes al emplear las TIC en el proceso de E-A

Finalmente, conviene resaltar que más de la mitad de los tutores encuestados $(56,4 \%)$ cree que necesita bastante formación en relación a la implementación de estrategias metodológicas activas al emplear las TIC en el aula, mientras que el $27 \%$ considera que necesita mucha más formación en este aspecto. Tan solo el $13,5 \%$ de los maestros participantes en este estudio cree que necesita recibir poca formación más en esta temática, mientras que cerca del $2,3 \%$ considera no necesitar nada de formación.

\section{DISCUSIÓN Y CONCLUSIONES}

El presente estudio revela, en primer lugar, que la mayoría de los maestros participantes en el mismo creen que su grado de dominio de las TIC es bueno y que la presencia de las TIC en el proceso de enseñanza-aprendizaje es esencial. Este resultado coincide con una de las conclusiones que se obtienen en la investigación de Céspedes (2017), en la que se expone que la mayoría del profesorado de Primaria de la Región de Murcia considera que el uso de las TIC en el ámbito educativo es esencial para preparar al alumnado del siglo XXI.

Con respecto al primer objetivo general de esta investigación, cabe concluir que los maestros tutores encuestados utilizan con mayor frecuencia, en el proceso de enseñanzaaprendizaje, recursos digitales y dispositivos menos innovadores a nivel pedagógico, como son los libros de texto digitales, los vídeos o las aplicaciones ofimáticas. A su vez, estos docentes introducen en el aula con menor frecuencia aquellos dispositivos y recursos más innovadores, como las tabletas o la realidad virtual, entre otros.

En concreto, entre los recursos digitales y tecnológicos que los docentes incorporan con más frecuencia en el proceso de enseñanza-aprendizaje se encuentran el ordenador, los proyectores y las pizarras digitales interactivas, lo que evidencia la progresiva incorporación de estos dispositivos en gran parte de los centros educativos de la Región de Murcia. En relación a lo anterior, cabe destacar que la presencia de las pizarras digitales interactivas en las aulas está teniendo una valoración positiva por la mayoría de los docentes en España (Domingo \& Marquès, 2013). 
Otro recurso empleado con cierta frecuencia por los maestros encuestados es el libro de texto digital. Esto último puede ser debido, entre otros aspectos, al impulso de programas gratuitos que contribuyen a la incorporación de estos libros digitales en los centros de enseñanza de las distintas comunidades autónomas (Mato \& Álvarez, 2019).

Cabe resaltar, por otra parte, que la mayoría de los maestros tutores encuestados no incorpora nunca las tabletas en el proceso de enseñanza-aprendizaje o las incorpora ocasionalmente, a pesar de que estos dispositivos se consideran importantes en la enseñanza básica, debido a las facilidades que reúnen para potenciar el aprendizaje y la competencia digital del alumnado (Fuentes et al., 2019).

Por otro lado, se ha comprobado como algunas tendencias emergentes en el ámbito de la investigación educativa, como son la realidad aumentada, la robótica educativa y la realidad virtual, son las menos empleadas en el proceso de enseñanza-aprendizaje desarrollado por la mayoría de los docentes participantes en este estudio.

En relación a lo anterior, se debe tener en cuenta que el nivel de dominio que poseen los maestros en el uso de las TIC influye en la frecuencia con la que los mismos incorporan recursos digitales y tecnológicos en el aula, ya que se ha demostrado que aquellos que contaban con un mayor nivel de dominio de las TIC ponían en práctica con más frecuencia, en el proceso de enseñanza-aprendizaje, recursos como las tabletas, los teléfonos móviles inteligentes, los recursos multimedia gamificadores, las aplicaciones de internet, los blogs, la realidad aumentada y la robótica, entre otros.

Asimismo, este estudio revela que la experiencia docente es también un factor influyente en la frecuencia de uso de algunos recursos TIC más innovadores. En este caso, se observa que los que han trabajado más años como maestros se decantan menos por el empleo de recursos emergentes en el proceso de enseñanza-aprendizaje, como las tabletas y los recursos multimedia gamificadores, ya que los incorporan en el aula con menor frecuencia que aquellos que cuentan con menos años de experiencia docente.

Conviene tener en cuenta, además, que la baja frecuencia con la que los docentes encuestados utilizan recursos como la robótica o las tabletas puede venir determinada, entre otros factores, por la falta de este tipo de dispositivos o recursos en los centros educativos. En esta línea, se debe señalar que el número y calidad de los recursos digitales que se usen en los colegios influyen las políticas educativas que se hayan impulsado con respecto a la distribución de los materiales y herramientas digitales en los centros de enseñanza (Sanabria et al., 2017).

Por otra parte, cabe resaltar que, de los recursos tecnológicos y digitales propuestos por los propios maestros en el cuestionario, uno de los que más se ha repetido es el uso de plataformas educativas, como Google Classroom. Estas últimas permiten al docente desarrollar y organizar el proceso de enseñanza de forma virtual.

Con respecto a este último resultado, es necesario destacar que en el momento en el que los maestros contestaron al cuestionario de este estudio, las clases en los colegios de Educación Primaria de toda España estaban suspendidas, ya que el país se encontraba en confinamiento por el estado de alarma que se había establecido con motivo del COVID-19. En este sentido, una de las razones por las que una de las respuestas que más se repitió en el cuestionario fue el uso de plataformas como Google Classroom pudo ser que, en el momento en el que respondieron a este cuestionario, algunos de los docentes encuestados se encontraban empleando estos recursos para desarrollar el proceso de enseñanza-aprendizaje con sus estudiantes de forma telemática durante el estado de alarma.

En relación al segundo objetivo de esta investigación, podemos concluir que los maestros participantes en la investigación suelen incluir, en más de una ocasión, metodologías activas en el proceso de enseñanza-aprendizaje a la hora de hacer uso de las TIC, ya que los resultados evidencian que el aprendizaje cooperativo, el aprendizaje basado en proyectos y el aprendizaje basado en juegos son estrategias metodológicas que se emplean con cierta frecuencia.

Sin embargo, la gamificación y el flipped classroom, a pesar de ser metodologías activas e innovadoras en relación al uso de las TIC, son utilizadas con poca frecuencia por los docentes encuestados. En concreto, de las metodologías que se plantearon en esta investigación, el 
flipped classroom es la menos utilizada por los maestros, a pesar de que numerosas investigaciones recomiendan la implementación de esta metodología ya que, además de fomentar el uso de las TIC, favorece la interacción entre el alumnado y el profesorado; fomenta la colaboración en el aula; facilita la implicación de las familias en el proceso de aprendizaje del alumnado; y permite al personal docente atender mejor a las necesidades de los estudiantes (Bergmann \& Sams, 2012; Tourón \& Santiago, 2015).

Con respecto a lo anterior, es importante tener en cuenta que, de los docentes participantes en el estudio, los que cuentan con mayor nivel de dominio de las TIC son los que implementan con más frecuencia en el aula la gamificación y el flipped classroom. Asimismo, conviene resaltar que aquellos que contaban con más experiencia docente son empleaban con menos frecuencia la gamificación.

Al respecto, cabe concluir que el nivel de dominio de las TIC y la experiencia docente que los maestros poseen influyen en su decisión de implementar en el aula o no tendencias metodológicas emergentes en el uso de las TIC.

Otro de los aspectos que se han analizado es la percepción que tiene el profesorado sobre la implementación de metodologías activas a la hora de utilizar las TIC en el proceso de enseñanza-aprendizaje, ya que la adquisición o mejora de los aprendizajes por parte del alumnado no depende tanto de las tecnologías digitales empleadas, sino de las estrategias metodológicas o didácticas que se pongan en práctica haciendo uso de las mismas (Cabero, 2001; Llorente et al. 2015; Nieto \& Marquès, 2015). En relación a esto, concluimos que, aunque son pocos los docentes que consideran que la metodología empleada constituya el elemento más relevante, la mayoría ha tenido en cuenta la importancia de la misma de cara al uso de las TIC en el aula.

Por otro lado, el profesorado participante considera que necesita una mayor formación en relación empleo de estrategias metodológicas activas a la hora de utilizar las TIC en el proceso de enseñanza-aprendizaje. Este resultado es similar al obtenido en la investigación de Céspedes (2017), en la que se concluye que los maestros de Educación Primaria de la Región de Murcia consideran que hace falta formación, tanto de cara a la utilización de las TIC como a nivel metodológico.

Al respecto, es importante analizar y estudiar si se debe incrementar la formación del personal docente en relación al uso pedagógico de las TIC, ya que la falta de formación del profesorado en la implementación de estrategias metodológicas centradas en el estudiante dificulta la innovación (Moreno, 2018). A su vez, la falta de actualización de los docentes en relación al uso de las TIC a nivel instrumental y metodológico dificulta la puesta en práctica de las mismas en el ámbito educativo (Colás et al., 2018).

En relación a los logros alcanzados con el desarrollo de la presente investigación, cabe destacar que la misma ha permitido identificar cuáles suelen ser los recursos digitales y tecnológicos que la muestra obtenida de docentes tutores de Educación Primaria de la Región de Murcia, en concreto, suele incorporar en el proceso de enseñanza-aprendizaje con mayor y menor frecuencia. Asimismo, a través del estudio, se ha comprobado cuáles son las estrategias metodológicas activas implementadas por estos maestros, así como cuál es la percepción de los mismos con respecto al uso de este tipo de estrategias al hacer uso de las TIC en el aula.

Por otro lado, este estudio permite identificar, en términos generales, cuál es el nivel de dominio de las TIC de los docentes participantes, así como la formación qué poseen y/o precisan los mismos en relación al uso pedagógico de las tecnologías digitales.

Con respecto a las limitaciones de esta investigación, por otra parte, cabe destacar que el tratamiento y análisis de datos desarrollado en la misma es cuantitativo y, aunque desde este enfoque se han alcanzado los objetivos de la investigación, con la introducción de técnicas o procedimientos de análisis y tratamiento cualitativo de los datos, posiblemente, se hubiera podido ampliar o enriquecer los resultados obtenidos.

Otra de las limitaciones de este estudio ha sido que no se han recogido evidencias explícitas sobre los recursos tecnológicos con los que cuentan, actualmente, los centros educativos en los que trabajan los maestros tutores participantes. 
De cara al futuro, otras posibles líneas de investigación podrían estar relacionadas con el análisis de los recursos digitales y tecnológicos disponibles en estos centros, así como de los planes específicos y de las estrategias que se desarrollan en los mismos en relación al uso de las TIC en el proceso de enseñanza-aprendizaje. A su vez, se pueden llevar a cabo estudios en los que se analice el tipo de actividades o prácticas educativas concretas que desarrollan los docentes en esta etapa educativa a través de técnicas cualitativas para, de esta manera, ampliar los resultados obtenidos en la presente investigación.

\section{ENLACES}

https://bit.ly/3kPmX6r

\section{REFERENCIAS BIBLIOGRÁFICAS}

Aznar. I., Romero, J.M., \& Rodríguez, A.M. (2018). La tecnología móvil de Realidad Virtual en educación: una revisión del estado de la literatura científica en España. EDMETIC, Revista de Educación Mediática y TIC, 7(1), 256-274. https://doi.org/10.21071/edmetic.v7i1.10139

Bergmann, J. \& Sams, A. (2012). Flip your classroom. Reach Every Student in Every Class Every Day. ITSE.

Boyle, S. (2011). Teaching Toolkit: and introduction to games based learning. https://bit.ly/307gsW0

Bravo, F.A. \& Forero, A. (2012). La robótica como un recurso para facilitar el aprendizaje y desarrollo de competencias generales. Teoría de la Educación. Educación y Cultura en la Sociedad de la Información, 13(2), 120-136. https://bit.ly/2A34QbL

Cabero, J. (2001). Tecnología Educativa. Diseño y utilización de medios en la enseñanza. Paidós.

Cabero, J. \& Martínez, A. (2019). Las tecnologías de la información y la comunicación y la formación inicial de los docentes: modelos y competencias digitales. Profesorado: Revista de currículum y formación del profesorado, 23(3), 247-268. https://doi.org/10.30827/profesorado.v23i3.9421

Cabero, J. \& Ruiz, J. (2018). Las Tecnologías de la información y la comunicación para la inclusión: reformulando la brecha digital. International Journal of Educational Research and Innovation, 9, 16-30. https://bit.ly/2MpOZH3

Cardona, M.C. (2002). Introducción a los Métodos de Investigación en Educación. EOS, Instituto de Orientación Psicológica Asociados.

Céspedes, R. (2017). La integración de las tecnologías de la información y la comunicación (TIC) en los centros de Educación Primaria de la Región de Murcia. (Tesis doctoral, Universidad de Murcia). Universidad de Murcia. https://bit.ly/2U9anVk

Colás, M.P., De Pablos, J. \& Ballesta, J. (2018). Incidencia de las TIC en la enseñanza en el sistema educativo español: una revisión de la investigación. Revista de Educación a Distancia, 56(2). https://bit.ly/3cAqDVs

Díaz-Barriga, A. (2013). TIC en el trabajo del aula. Impacto en la planeación didáctica. Revista Iberoamericana de Educación Superior (RIES), 4(10), 3-21. https://doi.org/10.22201/iisue.20072872e.2013.10.88

Domingo, M. \& Marquès, P. (2011). Aulas 2.0 y uso de las TIC en la práctica docente. Comunicar, 19(37), 169-175. https://doi.org/10.3916/C37-2011-03-09

Domingo, M. \& Marquès, P. (2013). Experimentación del uso didáctico de la pizarra digital interactiva (PDI) en el aula: plan formativo y resultados. Enseñanza \& Teaching, 31(1), 91-108. https://bit.ly/2ACXwUq 
Fuentes, J., Albertos, J. \& Torrano, F. (2019). Towards the Mobile-Learning in the School: Analysis of Critical Factors on the Use of Tablets in Spanish. Education in the Knowledge Society, 20. https://doi.org/10.14201/eks201920 a3

González, E. \& Zariquiey, F. (2012). Las TIC y el aprendizaje cooperativo. En J.C. Torrego y A. Negro (Coords.), Aprendizaje cooperativo en las aulas. Fundamentos y recursos para su implantación (pp.207-238). Alianza Editorial.

Granados, A. (2015). Las TIC en la enseñanza de los métodos numéricos. Sophia Educación, 11(2), 143-154. https://bit.ly/3cyP71x

Hernández, R., Fernández, C. y Baptista, P. (2014). Metodología de la investigación. McGrawHill.

Hernández, R.M. (2017). Impacto de las TIC en la educación: Retos y perspectivas. Propósitos y Representaciones, 5(1), 325-347. http://doi.org/10.20511/pyr2017.v5n1.149

Llorente, M.C., Barroso, J. \& Cabero, J. (2015). Las tecnologías de la información y la comunicación: principios para su aplicación, integración y selección educativa. En J. Cabero, y J. Barroso (Eds.). Nuevos retos en tecnología educativa (pp.41-68). Síntesis.

López, J., Pozo, S. \& Fuentes, A. (2020). La realidad de la aplicación de las redes sociales en el entorno educativo. El caso de una cooperativa de enseñanza de Ceuta. Revista Electrónica Educare, 24(1), 1-22. https://doi.org/10.15359/ree.24-1.17

Martínez, S., Gutiérrez, J.J. \& Fernández, B. (2018). Percepciones y uso de las TIC en las aulas inclusivas: Un estudio de caso. EDMETIC. Revista de Educación Mediática y TIC, 7(1), 87-106. https://doi.org/10.21071/edmetic.v7i1.10132

Mato, D. \& Álvarez, D. (2019). La implementación de TIC y MDD en la práctica docente de Educación Primaria. Campus Virtuales, 8(2), 73-84. https://bit.ly/2MpoO3c

Moreno, J.A. (2018). Las tic en el proceso de enseñanza y aprendizaje. En M.L. Cacheiro (Ed.), Educación y tecnología: estrategias didácticas para la intervención de las TIC (pp.9-34). UNED. https://bit.ly/2XawgRU

Nieto, E. \& Marquès, P. (2015). La mejora del aprendizaje a través de las nuevas tecnologías y de la implantación del currículo bimodal. Multiárea. Revista de didáctica, 7, 7-30. https://doi.org/10.18239/mard.v0i7.692

Recio, S. (2019). Experiencias robóticas en Infantil. Revista Interuniversitaria de Investigación en Tecnología Educativa, 7, 73-84. https://doi.org/10.6018/riite.399641

Rekalde, I. \& García, J. (2015). El Aprendizaje Basado en Proyectos: un constante desafío. Innovación educativa, 25, 219-234. https://doi.org/10.15304/ie.25.2304

Sanabria, A.L., Álvarez, Q. \& Peirats, J. (2017). Las políticas educativas en la producción y distribución de materiales didácticos digitales. RELATEC: Revista Latinoamericana de Tecnología Educativa, 16(2), 63-77. https://doi.org/10.17398/1695-288X.16.2.63

Sánchez, J., Ruíz, J. \& Sánchez, E. (2017). Flipped classroom: claves para su puesta en práctica. EDMETIC, Revista de Educación Mediática y TIC, 6(2), 336-358. https://doi.org/10.21071/edmetic.v6i2.5832

Sebastian, R., Olanda, R. \& Orduña, J.M. (2013). Introducción de metodologías de aprendizaje basado en problemas en el marco de las TIC. En M. Marqués, J.M. Badía y S. Barrachina (Eds.), Actas de las XIX Jornadas sobre la Enseñanza Universitaria de la Informática (pp.153-160). Publicacions de la Universitat Jaume I. http://doi.org/10.6035/eTliT.2013.13

Tourón, J. \& Santiago, R. (2015). El modelo Flipped Learning y el desarrollo del talento en la escuela. Revista de Educación, 368, 33-65. https://doi.org/10.4438/1988-592X-RE-2015$\underline{368-288}$

Villena, T., Cózar, R. \& Gónzález-Calero, J.A. (2018). Gamificación en grupos interactivos para enseñar y aprender Historia. Una experiencia en Educación Primaria. En J. Monteagudo, 
A. Escribano \& C.J. Gómez (Eds.), Educación histórica y competencias transversales: narrativas, TIC y competencia lingüística (pp. 275-286). Editum. https://bit.ly/2YebEvx

\section{INFORMACIÓN SOBRE EL AUTOR}

\section{David Castillo López}

Universidad de Murcia

Graduado Universitario en Educación Primaria, con mención en Necesidades Específicas de Apoyo Educativo por la Universidad de Murcia. Máster en Tecnología Educativa: e-learning y gestión del conocimiento por la Universidad de Murcia. Maestro interino de Educación Primaria en la especialidad de Pedagogía Terapéutica.

Líneas de interés de investigación: las TIC en Educación Primaria; metodologías emergentes en relación a las TIC.

\section{(c)}

Los textos publicados en esta revista están sujetos a una licencia de Reconocimiento 4.0 España de Creative Commons. Puede copiarlos, distribuirlos, comunicarlos públicamente y hacer obras derivadas siempre que reconozca los créditos de las obras (autoría, nombre de la revista, institución editora) de la manera especificada por los autores o por la revista. La licencia completa se puede consultar en:Licencia Creative Commons Atribución-NoComercial-Compartir por igual 4.0 Internacional. 